\section{TRANSPARENCIA Y ACCESO A LA INFORMACIÓN EN SEDES ELECTRÓNICAS MUNICIPALES}

\author{
Marta Pagán Martínez \\ José Vicente Rodríguez Muñoz \\ Ricardo César Gonçalves Sant'Ana ${ }^{* * *}$ \\ Wagner Luiz Lourenzanit*t+
}

RESUMEN El propósito de esta investigación fue analizar el nivel de consolidación y situación actual de los servicios electrónicos ofrecidos por 64 Ayuntamientos de la provincia de Albacete a través de sus sedes web oficiales. Para ello, se definieron y seleccionaron los servicios básicos online que los ayuntamientos debían ofrecer a los ciudadanos, siguiendo las directrices establecidas en el Plan de Acción de la eEurope 2005 y en el Plan de Actuación i2010. Los datos fueron recolectados durante 12 meses, usando una metodología de evaluación del portales web y servicios online ofrecidos por los ayuntamientos evaluados a partir de unos indicadores. Los instrumentos de recolección de datos fueron el análisis documental, cuestionarios y observación experimental. Los resultados reflejan el esfuerzo de los ayuntamientos por mejorar el diseño de sus webs y los servicios electrónicos ofrecidos a los ciudadanos, aunque todavía queda un largo camino por recorrer hasta alcanzar los parámetros marcados por la Unión Europea.

Palabras Clave: Administración electrónica. Gobierno electrónico.
$\begin{aligned} & \text { Administración local. Evaluación web. Sociedad de la } \\ & \text { información. }\end{aligned}$

\footnotetext{
* Doutora em Métodos y Técnicas Actuales en Información y Documentación pela Universidad de Murcia, espanha. Docente no Departamento de Información y Documentación da Universidad de Murcia, Espanha. E-mail: pagan.marta@gmail.com.

** Doutor em Informática pela Universidad de Murcia, Espanha. Docente do Departamento de Información y Documentación da Universidad de Murcia, Espanha. Email: jovi@um.es.

*** Doutor em Ciência da Informação pela Universidade Estadual Paulista Júlio de Mesquita Filho, Brasil. Docente permanente no Programa de Pós-Graduação em Ciência da Informação da Universidade Estadual Paulista Júlio de Mesquita Filho, Brasil.

Email: ricardosantana@tupa.unesp.br.

***** Doutor em Engenharia de Produção pela Universidade Federal de São Carlos, Brasil. Profesor do Departamento de Administração da Facultade de Ciências e Engenharia da Universidade Estadual Paulista Júlio de Mesquita Filho, Brasil.

Email: wagner@tupa.unesp.br.
}

\section{INTRODUCCIÓN}

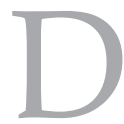
ebido al enorme impacto que tienen actualmente en nuestra sociedad las Nuevas Tecnologías de la Información y la Comunicación (TIC), la sociedad debe adaptar todas sus actuaciones y estrategias a las nuevas necesidades que van surgiendo. Deben adaptarse las infraestructuras de comunicaciones, los procesos de aprendizaje, las personas y los esquemas con los que poder gestionar la información lo más eficientemente posible para poder afrontar los cambios.

Las TIC se han convertido, en una importante herramienta para el proceso de reforma, mejora y actualización de la gestión pública, permitiendo así una mayor satisfacción de los ciudadanos en la prestación de servicios públicos y una mejora en su participación a la hora de elaborar políticas públicas (OCDE, 2003).

La introducción de las TIC en la esfera de la Administración Pública ha implicado notables cambios en la gestión de sus recursos de información y permite que la información sea procesada y compartida de un modo diferente.

Gracias a las TIC, las empresas y los ciudadanos pueden interactuar con las Administraciones Públicas (AAPP) y éstas entre sí, así como compartir y distribuir cualquier información de forma instantánea desde cualquier lugar del mundo y de formas muy diversas. 
Ahora, tanto ciudadanos como profesionales de la información y de la documentación deben adaptarse a los nuevos cambios, ya que las TIC implican una importante transformación en el modo de comunicarse con los organismos públicos.

La Administración Pública española está llevando a cabo diversas iniciativas e incorporando las herramientas necesarias para sumarse a la actual Sociedad de la Información y el Conocimiento. Por ello, es evidente que la Administración Pública desempeña un papel fundamental para fomentar e impulsar el desarrollo y la construcción de dicha sociedad. Es más, una de las herramientas que posee la Administración Pública para favorecer el desarrollo de la Sociedad de la Información, es ofrecer servicios públicos en línea, esto es lo que se conoce como: "e-Administración", "Administración electrónica" o "Administración en línea". Con lo que queda de manifiesto que Internet es el protagonista hoy día en todos los ámbitos de la vida, principalmente para facilitar el acceso de los ciudadanos a la Administración, pues la clave es lograr una Administración electrónica (Ae) orientada al usuario o ciudadano.

Los ayuntamientos son conscientes de la importancia de las TIC como instrumento de ayuda, por lo que se están esforzando por modernizar su Administración Local y ofrecer más y mejores servicios a sus ciudadanos.

Por todo ello y partiendo de la idea de que los ayuntamientos son la Administración Pública más próxima a los ciudadanos, la finalidad de este estudio ha sido la de analizar y evaluar las sedes webs oficiales de los Ayuntamientos de la provincia de Albacete, para conocer su nivel de adaptación a la Ley 11/2007, de 22 de junio, de Acceso Electrónico de los Ciudadanos a los Servicios Públicos (LAECSP), el nivel de transparencia, nivel de accesibilidad, nivel de informatización de los servicios ofrecidos y nivel de madurez de los servicios electrónicos ofrecidos por los ayuntamientos objeto de estudio. Así, como conocer qué tipos de servicios de información online ofrecen los ayuntamientos y los flujos de información entre administración-ciudadano que se establecen a través de sus procedimientos Web.

\section{MARCO CONTEXTUAL: estudios previos $y$ antecedentes}

Tras hacer una revisión bibliográfica para conocer el estado de la cuestión se observó que uno de los principales problemas encontrados fue la escasa cantidad de estudios de este tipo y la ausencia de estudios al respecto centrados en la Comunidad Autónoma de Castilla-La Mancha y más concretamente, de la provincia de Albacete, teniendo en cuenta, además, que la información sobre el tema que nos ocupa está en continuo cambio y actualización, por lo que ha de ser revisada constantemente.

La mayor cantidad de estudios españoles encontrados son referentes a las Islas Baleares, Andalucía y la Región de Murcia, entre otros.

Asimismo, la Organización para la Cooperación y el Desarrollo Económico (OCDE) ha generado una notable cantidad de estudios y documentos sobre Administración Electrónica, algunos de los cuales han sido de capital importancia para el desarrollo del presente estudio. Así, cabe señalar el estudio de la OCDE (2005), que fue traducido por el Instituto Nacional de Administración Pública (INAP, 2009).

Por otro lado, destaca la adaptación de la Administración al nuevo entorno en el que priman: las nuevas tecnologías, la interrelación entre personas, los procesos, las leyes y las normas, que ha implicado el surgimiento de estudios en los que se analizan diversos aspectos, tales como: los problemas de identificación de los usuarios ante servicios que requieren autentificación; la participación ciudadana que incumbe al proceso democrático o la propia estructuración de la información no siempre es adecuada; las fases de ajuste del sector público a esta nueva realidad; etc.

Como antecedente al presente estudio destaca también el "Libro Verde" de la COMISIÓN EUROPEA (1998) que señala que los servicios del gobierno electrónico pueden desglosarse generalmente según las tres funciones principales que cumplen: Servicios de información para recoger la información ordenada y clasificada que se solicite (como los sitios web); Servicios de comunicación para la interacción entre individuos (particulares o empresas) o grupos de personas (como el correo electrónico o foros de discusión); y servicios de transacción para adquirir productos o servicios en línea o bien para presentar datos (como formularios públicos o escrutinios). 
De igual modo, cabe mencionar el "Plan de Acción eEurope 2002" (COMISIÓN EUROPEA, 2001) donde se apunta que una sociedad de la información para todos se acompaña de un proceso de evaluación comparativa para evaluar los progresos de los Estados miembros de la Unión Europea (UE, 2000). Este Plan de Acción establece una serie de líneas maestras o ejes de actuación, tales como: Una Internet más rápida, barata y segura con acceso a Internet más rápido y barato, con una Internet más rápida para investigadores y estudiantes, y con redes seguras y tarjetas inteligentes; Invertir en las personas y en formación para facilitar el acceso de la juventud europea a la era digital, para trabajar dentro de una economía basada en el conocimiento y para fomentar la anticipación de todos en una economía basada en el conocimiento; Estimular el uso de Internet para acelerar el comercio electrónico, para lograr una Administración en línea que ofrezca acceso electrónico a los servicios públicos, para la sanidad online, para contenidos digitales europeos, para las redes mundiales y para desarrollar sistemas de transporte inteligentes.

Otro antecedente es el "Plan de Acción de la eEurope 2005" (COMISIÓN EUROPEA, 2002) cuyo objetivo general es estimular el desarrollo de servicios, aplicaciones y contenidos, acelerando al mismo tiempo el despliegue de un acceso seguro a la Internet de banda ancha. El acceso de banda ancha se caracteriza por la alta velocidad y el acceso permanente a Internet. Existe además un objetivo transversal de acceso para todos con el fin de luchar contra la exclusión social, de que esté vinculada a necesidades especiales, a una discapacidad, a la edad o a la enfermedad. La eEurope 2005 sigue el enfoque, instaurado por la eEurope 2002, consistente en definir objetivos claros y efectuar una evaluación comparativa de los progresos conseguidos en su realización. Se trata de acelerar la adopción de nuevos instrumentos legislativos y de reorientar los programas existentes hacia las prioridades así definidas.

Por último, cabe destacar el "Plan de Actuación i2010" (COMISIÓN EUROPEA, 2006) que establece cinco de objetivos en materia de administración electrónica: 1) Ningún ciudadano debe quedarse atrás: promover la inclusión a través de la administración electrónica de manera que para 2010 todos los ciudadanos se beneficien de unos servicios de confianza e innovadores, así como de un acceso sencillo para todos; 2) La eficiencia y la eficacia deben hacerse realidad, contribuyendo de forma significativa, para 2010, a la satisfacción de los usuarios, a la transparencia y la rendición de cuentas, al aligeramiento de la carga administrativa y a la mejora de la eficiencia; 3) Implantar servicios clave de gran repercusión para los ciudadanos y las empresas, de manera que para 2010, el 100\% de la contratación pública esté disponible en forma electrónica, alcanzando el uso real el 50\%, con un acuerdo de cooperación sobre otros servicios en línea al ciudadano de gran repercusión; 4) Establecer las herramientas clave, que permitan a ciudadanos y empresas beneficiarse, para 2010, de un acceso autenticado cómodo, seguro e interoperable a los servicios públicos en toda Europa; 5) Fortalecer la participación y la adopción de decisiones democráticas, demostrando para 2010 unas herramientas que permitan el debate y la participación del público efectivos en la adopción de decisiones democráticas.

\section{OBJETIVOS}

Los objetivos que se pretendieron alcanzar con este estudio son los siguientes:

1. Analizar y evaluar los servicios ofrecidos por los Ayuntamientos de la Provincia de Albacete a través de sus sedes web oficiales.

2. Examinar qué ayuntamientos poseen sede web oficial, teniendo en cuenta el número de habitantes de cada municipio; saber cuáles son los que tiene dominio web propio y qué tipo de dominio (".es", ".org", ".net", ".com", ".info", etc.)

3. Comprobar el nivel de inserción de las TIC como herramienta para desarrollar un nuevo Sistema de Información en la Administración local más eficiente, y constatar el interés de los responsables políticos en cada momento por mantenerlos y mejorarlos, permitiendo conocer la evolución, en el estadio inicial de desarrollo, de esta aplicación tecnológica.

\section{PROCEDIMIENTOS METODOLÓGICOS}

El esquema metodológico de investigación se estructuró en cinco fases: 1. Recopilación bibliográfica sobre el tema objeto de 
investigación; 2. Determinación del universo a evaluar; 3. Selección de los indicadores de evaluación; 4. Análisis y evaluación de las sedes web objeto de estudio, estableciendo un marco evaluativo común para todos los servicios, que permita precisar el estado de informatización de cada uno de los servicios y el tipo de servicios ofrecidos; 5. Proceso de recogida de datos; 6. Análisis y proyección de los resultados.

\section{I Procedimiento para la recolección de datos}

Los datos fueron recolectados durante un período de 12 meses, usando una metodología exploratoria de análisis y evaluación de los portales web y servicios electrónicos ofrecidos por los ayuntamientos objeto de estudio a partir de la elaboración de indicadores de evaluación. Los instrumentos utilizados para la recolección de datos fueron el análisis documental, cuestionarios y observación experimental. Los cuestionarios para cada web están compuesto por 8 variables o grupos de indicares que a su vez constaban de un total de 54 indicadores para evaluar la información ofrecida por 64 ayuntamientos a través de sus portales web oficiales.

\subsection{Procedimientos para el análisis y evaluación}

A) Selección de indicadores para la evaluación de sedes web municipales

Para analizar y evaluar las sedes web de los ayuntamientos de la provincia de Albacete fue preciso seleccionar tanto los indicadores más adecuado y oportunos, en base a los parámetros marcados por la Unión Europea (UE), como la metodología de evaluación a seguir. Dicha selección se llevó a cabo a partir de diversas propuestas y evaluaciones realizadas en España y otros países, algunas de ellas son: SI-Loc@1 (2002), Moya (2003), West (2003), West (2004), Sureda y Comas (2004), Fundación AUNA (2005), estudios publicados en el Observatorio de la Región de Murcia por Gregorio Moya y su grupo de investigación (MOYA et al., 2005, 2006, 2009 y 2011) y los estudios la OCDE (2005) y el INAP (2009)
Tras analizar estas metodologías de evaluación, se escogió la utilizada por Gregorio Moya et al. (2005, 2006, 2009 y 2011), que han servido como guía, modelo y base para el presente estudio, por parecernos la más contrastada, pertinente y por ser la que forma parte del equipo de investigación de donde emerge la presente investigación. Por otro lado, es la que se adecua mejor al ámbito de estudio que aquí nos ocupa.

Por consiguiente, aunque se añadieron indicadores a este estudio, no se ha pretendido desarrollar una metodología o indicadores nuevos, sino que se buscaba ajustarse a los estándares ya establecidos por la UE y emplear unos indicadores ya consolidados y probados, para la consecución de unos resultados fehacientes.

Tras analizar diversas alternativas para reflejar con mayor veracidad y exactitud la realidad de las distintas sedes web municipales, se ha escogido un total de 54 indicadores que se agrupan bajo ocho conceptos sobre los se ha trabajado para recabar la máxima información sobre cada portal web evaluado, puesto que suelen abarcar la mayoría de los contenidos ha de incluir una web municipal. Los ocho grupos de indicadores son:

I. Aspectos formales.

II. Funcionalidad.

III. El Ayuntamiento.

IV. La Ciudad o Municipio.

V. Información para los Ciudadanos

VI. Información Turística y de Ocio.

VII. Información sobre las Empresas.

VIII. Servicios de Participación Ciudadana.

Estos grupos de indicadores sirven para evaluar los contenidos de carácter informativo. Por lo tanto, se ha llegado a la conclusión de que es suficiente con valorar la presencia o ausencia de los contenidos en cada una de las webs municipales analizadas y evaluadas. Así, salvo con la excepción del indicador 14 que se mide mediante un promedio de número de clic, al resto de indicadores se les asignó el valor uno (1) para la respuesta positiva "SÍ" y valor cero (0) para la respuesta negativa "NO". A continuación se muestran las tablas con los ocho grupos de indicadores o servicios públicos básicos online que las web deberían ofrecer a la ciudadanía. 
La Tabla 1 muestra el primer grupo donde se han incluido aquellos indicadores que permiten identificar con claridad la autoría y responsabilidad de la página web, la forma en que el usuario puede contactar con los responsables de la misma (en este caso, serían los ayuntamientos) y si se especifica cuándo se ha realizado la última actualización de la web.

Tabla 1: Indicadores sobre aspectos formales.

\section{L.Aspectos Formales}

\section{Indicadores}

\begin{tabular}{cll}
\hline 1. & $\begin{array}{l}\text { ¿La autoría de la web está } \\
\text { claramente definida por el } \\
\text { Ayuntamiento? }\end{array}$ & SI/NO \\
\hline 2. & Indica la Dirección Postal & SI/NO \\
\hline 3. & $\begin{array}{l}\text { Indica el teléfono de contacto del } \\
\text { Ayuntamiento }\end{array}$ & SI/NO \\
\hline 4. & $\begin{array}{l}\text { Indica el e-mail del Ayuntamiento } \\
\text { o del webmaster }\end{array}$ & SI/NO \\
\hline 5. & $\begin{array}{l}\text { Indica cuándo ha sido la última } \\
\text { actualización en la home page o } \\
\text { página de Inicio }\end{array}$ & SI/NO \\
\hline
\end{tabular}

Fuente: Elaboración propia.

El bloque de indicadores de la Tabla 2 permite medir el grado de funcionalidad de los portales web a evaluar, es decir, permite medir los aspectos físicos como la mejora de la interfaz de usuario. Los indicadores 6 y 7 junto con el indicador 15 permiten conocer las facilidades de navegación en la web. Los indicadores 9 y 10 exhiben el grado de interacción de la web evaluada con otras sedes web, a través de los enlaces que existen hacia otras administraciones y de la descarga de utilidades que puedan ser necesarios para ver determinados documentos (plugins, visores, etc.)

El indicador 13 mide el nivel de accesibilidad web, ya que actualmente las webs de las Administraciones Públicas deben adaptarse a las normativas obligatoriamente. Para llevar a cabo la evaluación de este indicador, se aplicó el test TAW (Test Accesibilidad Web: http://www.tawdis.net), que mide el grado de accesibilidad según las normas WAI (Web Accessibility Initiative: http://www.w3.org/ WAI). El valor "NO" indica que la web no es accesible. Para que una web sea considerada como "accesible" ha de alcanzar en el test TAW el nivel AA que significa que es accesible en su nivel 2. Un valor A indica que es accesible a primer nivel, según el test TAW de accesibilidad; un valor AA indica que alcanza una accesibilidad de nivel 2; y el valor AAA indica el máximo grado de accesibilidad definido en el test TAW.

El indicador 14, llamado "profundidad" mide el promedio del número de clics necesarios hasta llegar a la información deseada y permite conocer tanto el grado de jerarquización de los contenidos como la complejidad de la web. Normalmente, se considera que en una web bien estructurada es posible encontrar y acceder a toda la información que alberga en 5 o menos clics. Por consiguiente, si en dicha web son necesarios más de 5 clics esto indicaría que la web tiene problemas de diseño o de estructura de los contenidos.

El indicador 16, permite conocer si en el portal web se incluye el menú conocido como Hilo de Ariadna, Migas de pan o Breadcrumbs, que son menús de ubicación que permite al usuario saber dónde se encuentra dentro del sitio web, así como regresar al punto de partida. De este modo se le brinda al sitio web un mayor nivel de usabilidad y permite situar al usuario de la web en el contexto del portal, indicando una posible ruta desde la página principal al documento actual.

Tabla 2 - Indicadores sobre la funcionalidad de la web

\section{Funcionalidad}

\section{Indicadores}

\begin{tabular}{cll}
\hline 6. & Mapa web & SI/NO \\
\hline 7. & $\begin{array}{l}\text { Buscador interno (búsquedas en } \\
\text { la propia web) }\end{array}$ & SI/NO \\
\hline 8. & Versiones en otros idiomas & SI/NO \\
\hline 9. & $\begin{array}{l}\text { Enlaces hacia otras } \\
\text { administraciones }\end{array}$ & SI/NO \\
\hline 10. & Descarga de utilidades & SI/NO \\
\hline 11. & $\begin{array}{l}\text { Resolución de pantalla para la } \\
\text { que está optimizada }\end{array}$ & SI/NO \\
\hline 12. & $\begin{array}{l}\text { Navegadores para los que está } \\
\text { optimizada }\end{array}$ & SI/NO \\
\hline 13. & $\begin{array}{l}\text { Cumple con las normas WAI } \\
\text { (Accesibilidad: AAA/AA/A/) }\end{array}$ & SI/NO \\
\hline 14. & Profundidad (Número de clics) & N $^{\circ}$ Clic \\
\hline 15. & $\begin{array}{l}\text { Se puede volver desde cualquier } \\
\text { página a la página de inicio de } \\
\text { la web }\end{array}$ & SI/NO \\
\hline 16. & $\begin{array}{l}\text { Posee una menú “migas de pan” } \\
\text { para facilitar la usabilidad }\end{array}$ & SI/NO \\
\hline
\end{tabular}

Fuente: Elaboración propia 
En la Tabla 3 muestra el grupo de indicadores que permiten evaluar la información institucional que ofrecen los ayuntamientos a través de sus sedes web.

Tabla 3: Indicadores sobre información relativa al Ayuntamiento

\section{Ш. ElAvuntamiento}

\section{Indicadores}

\begin{tabular}{|c|c|c|}
\hline & $\begin{array}{l}\text { Bienvenida del Alcalde/ } \\
\text { Alcaldesa }\end{array}$ & SI/NO \\
\hline 18. & Indica los Órganos de Gobierno & SI/NO \\
\hline 19. & $\begin{array}{l}\text { Grupos políticos que conforman } \\
\text { la Corporación Municipal o } \\
\text { en su caso, la composición } \\
\text { completa del Pleno Municipal. }\end{array}$ & SI/NO \\
\hline 20. & Actas de los Plenos Municipales & SI/NO \\
\hline 21. & Ordenanzas Municipales & SI/NO \\
\hline 22. & Calendario Fiscal Municipal & SI/NO \\
\hline 23. & Presupuestos Municipales & SI/NO \\
\hline 24. & $\begin{array}{l}\text { Directorio de Servicios } \\
\text { Municipales }\end{array}$ & SI/NO \\
\hline 25. & $\begin{array}{l}\text { Se puede consultar el Plan } \\
\text { de Ordenación urbana del } \\
\text { municipio }\end{array}$ & SI/NO \\
\hline
\end{tabular}

Fuente: Elaboración propia

El grupo de indicadores de la Tabla 4 mide la información existente en la sede web sobre aspectos generales del municipio (situación geográfica, historia del municipio, datos estadísticos sobre población, economía, etc.).

Tabla 4: Indicadores sobre información relativa a la ciudad o municipio

\section{LV.La Ciudad o Municipio}

\section{Indicadores}

\begin{tabular}{lll}
\hline 26. & $\begin{array}{l}\text { Situación geográfica del } \\
\text { municipio }\end{array}$ & SI/NO \\
\hline 27. & Historia del municipio & SI/NO \\
\hline 28. & $\begin{array}{l}\text { Recursos multimedia: galería } \\
\text { fotográfica, vídeos, webcams, } \\
\text { etc. }\end{array}$ & SI/NO \\
\hline 29. & $\begin{array}{l}\text { Incluye un apartado con los } \\
\text { principales datos estadísticos }\end{array}$ & SI/NO \\
\hline
\end{tabular}

Fuente: (PAGÁN, 2013)
El grupo de la Tabla 5 lo conforman aquellos indicadores que proporcionan información sobre las actividades cotidianas del municipio, que pueden ser de interés para los ciudadanos del mismo y sus posibles visitantes, tales como: noticias, agenda, servicios de urgencias, centros culturales, educativos, etc.

Tabla 5: Indicadores sobre información para la ciudadanía

\section{Información para los Ciudadanos}

\section{Indicadores}

\begin{tabular}{lll}
\hline 30. & $\begin{array}{l}\text { Noticias de actualidad del } \\
\text { municipio }\end{array}$ & SI/NO \\
\hline 31. & $\begin{array}{l}\text { Agenda de actividades } \\
\text { municipales }\end{array}$ & SI/NO \\
\hline 32. & $\begin{array}{l}\text { Oficina de Atención al } \\
\text { Ciudadano (S.A.C / O.A.C) }\end{array}$ & SI/NO \\
\hline 33. & $\begin{array}{l}\text { Directorio sobre servicios de } \\
\text { urgencias }\end{array}$ & SI/NO \\
\hline 34. & Directorio Cultural & SI/NO \\
\hline 35. & $\begin{array}{l}\text { Directorio de Centros } \\
\text { Educativos }\end{array}$ & SI/NO \\
\hline 36. & $\begin{array}{l}\text { Directorio de Centros } \\
\text { Sanitarios }\end{array}$ & SI/NO \\
\hline 37. & $\begin{array}{l}\text { Información sobre el } \\
\text { transporte público }\end{array}$ & SI/NO \\
\hline 38. & Callejero de la localidad & SI/NO \\
\hline 39. & $\begin{array}{l}\text { Directorio de asociaciones } \\
\text { vecinales, culturales, } \\
\text { deportivas, etc. }\end{array}$ \\
\hline 40. & $\begin{array}{l}\text { Posibilidad de conseguir } \\
\text { gratuitamente una cuenta de } \\
\text { correo electrónico }\end{array}$ \\
\hline
\end{tabular}

Fuente: Elaboración propia

En la Tabla 6 se presenta el grupo de indicadores que permiten analizar los contenidos informativos referentes a la información turística y de ocio del municipio, tales como: dónde comer, dónde dormir, cómo llegar, fiestas y tradiciones, etc. Este tipo de información no sólo es de interés para los visitantes o turistas de la localidad, sino también para los residentes del municipio. 
Tabla 6: Indicadores sobre información turística y de ocio

\section{Información Turística y de Ocio}

\begin{tabular}{|c|c|c|}
\hline \multicolumn{3}{|c|}{ Indicadores } \\
\hline & $\begin{array}{l}\text { Información sobre cómo llegar al } \\
\text { municipio }\end{array}$ & SI/NO \\
\hline 42. & $\begin{array}{l}\text { Directorio sobre restaurantes, } \\
\text { bares, lugares de ocio, etc. }\end{array}$ & SI/NO \\
\hline 43. & $\begin{array}{l}\text { Directorio de alojamientos de la } \\
\text { localidad }\end{array}$ & SI/NO \\
\hline 44. & $\begin{array}{l}\text { Información sobre la Oficina de } \\
\text { Turismo }\end{array}$ & SI/NO \\
\hline 45. & $\begin{array}{l}\text { Fiestas y tradiciones de la } \\
\text { localidad }\end{array}$ & SI/NO \\
\hline 46. & $\begin{array}{l}\text { Guía turística, principales } \\
\text { lugares de interés, etc. }\end{array}$ & SI/NO \\
\hline 47. & Gastronomía y productos típicos & SI/NO \\
\hline
\end{tabular}

Fuente: Elaboración propia

En el grupo séptimo (Tabla 7) se agrupan tres indicadores $(47,48$ y 49 ) que muestran la información existente sobre las empresas del municipio, los polígonos industriales y la celebración de ferias. Además de la información mencionada en este apartado, también podemos encontrar indicadores que pueden ser de gran utilidad para el mundo empresarial y comercial en otros apartados de esta evaluación.

Tabla 7: Indicadores sobre información relativas a las empresas de la ciudad

\section{VUI. Información sobre las Empresas}

\section{Indicadores}

\begin{tabular}{rlc}
\hline 48. & $\begin{array}{l}\text { Directorio empresarial y/o } \\
\text { comercial }\end{array}$ & SI/NO \\
\hline 49. & $\begin{array}{l}\text { Información sobre el polígono } \\
\text { industrial de la localidad }\end{array}$ & SI/NO \\
\hline 50. & $\begin{array}{l}\text { Información sobre las ferias que se } \\
\text { realicen en la localidad }\end{array}$ & SI/NO \\
\hline
\end{tabular}

Fuente: Elaboración propia

Por último, la Tabla 8 muestra el grupo de indicadores que permite evaluar la existencia de mecanismos que impulsen la interactividad e interacción entre el Ayuntamiento y sus ciudadanos. El indicador 52 permite obtener información sobre el tiempo que tardan en responder y valorar si la respuesta se adecua a la pregunta efectuada (descartando las respuestas de tipo automático o semi-automático).

Tabla 8: Indicadores sobre los servicios de participación ciudadana ofrecidos

\section{VUI. Servicios de Participación Ciudadana}

\section{Indicadores}

\begin{tabular}{lll}
\hline 51. & Buzón de quejas o sugerencias & SI/NO \\
\hline 52. & $\begin{array}{l}\text { Respondió a la pregunta } \\
\text { realizada: ¿Qué trámites debo } \\
\text { realizar para darme de alta en } \\
\text { el Padrón municipal? }\end{array}$ & SI/NO \\
\hline 53. $\begin{array}{l}\text { Mecanismos de participación } \\
\text { ciudadana (FAQ, encuestas, } \\
\text { foros, etc.) }\end{array}$ & SI/NO \\
\hline 54. $\begin{array}{l}\text { Solicitar cita previa al Alcalde } \\
\text { y/o resto de miembros de la } \\
\text { Corporación municipal }\end{array}$ & SI/NO \\
\hline
\end{tabular}

Fuente: Elaboración propia

B) La muestra y contexto geo-demográfico del universo a evaluar

La muestra o universo a evaluar fueron 67 ayuntamientos con sede web oficial de 87 que tienen la provincia de Albacete. Sin embargo, de 67 ayuntamientos con sede web oficial encontrados se evaluaron 64, puesto que el resto se hallaba en un estado muy incipiente de creación y desarrollo en el momento de la evaluación, no se podía acceder a su URL (Página Web no disponible) o bien su proceso de creación estaba en anteproyecto. El objeto de evaluación fueron los portales web oficiales de los ayuntamientos de la provincia de Albacete. La fecha de realización de la evaluación fue del 01/01/2012 al 19/02/2013. Todas las sedes web fueron analizadas desde tres navegadores: Internet Explorer 8, Google Chrome 21.01180 .89 y Firefox 3.6.13.

Albacete es una provincia española situada en la Comunidad Autónoma de 
Castilla-La Mancha. La provincia posee un total de 402.837 habitantes (según los datos oficiales sobre las cifras de población a 1 de enero de 2012 del Instituto Nacional de Estadística- (INE) cuenta con una superficie de $14.926 \mathrm{~km}^{2}$ y tiene una densidad de población de 26,99 habitantes por $\mathrm{km}^{2}$. Su capital y municipio más poblado es la ciudad de Albacete, que cuenta con 172.472 habitantes (según los datos oficiales sobre las cifras de población a 1 de enero de 2012 del INE). La Provincia de Albacete está formada por un total de 87 municipios, con la distribución por grupos de población que se muestra en la Tabla 9.

Tabla 9: Distribución de los municipios de la provincia de Albacete por tamaño y población

\begin{tabular}{|c|c|c|c|c|}
\hline \multicolumn{5}{|c|}{$\begin{array}{l}\text { DISTRIBUCIÓN DE LOS MUNICIPIOS DE LA PROVINCIA DE ALBACETE } \\
\text { POR PROVINCIA, TAMAÑO Y POBLACIÓN }\end{array}$} \\
\hline $\begin{array}{l}\text { TIPO DE MUNICIPIO } \\
\text { (Habitantes) }\end{array}$ & Municipios & $\begin{array}{l}\text { \% total } \\
\text { Región }\end{array}$ & Habitantes & $\begin{array}{l}\% \text { total } \\
\text { Región }\end{array}$ \\
\hline $\begin{array}{l}\text { Municipios con menos de } \\
5.001\end{array}$ & 79 & $91,00 \%$ & 105.326 & $26,0 \%$ \\
\hline $\begin{array}{l}\text { Municipios entre } 5.001 \text { y } \\
10.000\end{array}$ & 2 & $2,00 \%$ & 14.849 & $4,00 \%$ \\
\hline $\begin{array}{l}\text { Municipios entre } 10.001 \text { y } \\
20.000\end{array}$ & 2 & $2,00 \%$ & 26.971 & $7,00 \%$ \\
\hline $\begin{array}{l}\text { Municipios entre } 20.001 \text { y } \\
50.000\end{array}$ & 3 & $4,00 \%$ & 83.219 & $20,00 \%$ \\
\hline Municipios con más de 50.000 & 1 & $1,00 \%$ & 172.472 & $43,00 \%$ \\
\hline TOTAL & 87 & $100,00 \%$ & 402.837 & $100,00 \%$ \\
\hline
\end{tabular}

Fuente: Elaboración propia

C) Localización y selección de los portales web a evaluar

Para la selección de la muestra a evaluar se han seguido los siguientes pasos:

1. Se determinó el universo a evaluar, es decir, determinar qué sedes web formarán parte del estudio. El objetivo era evaluar los portales web oficiales de los ayuntamientos de la provincia de Albacete. Así, la primera tarea fue realizar un rastreo para saber cuántos municipios posee la provincia de Albacete y tras esto, averiguar, de entre los 87 ayuntamientos que hay en la provincia, cuántos tienen sede web oficial. Además, se consultaron los datos o cifras de población para 2009 ofrecidos por el INE.

2. Se elaboró un listado con los 87 municipios de la provincia de Albacete en el que se incluye: nombre y dirección del Ayuntamiento, datos de contacto y enlace web (si lo hubiese).

3. Se contactó, por vía telefónica, con los 87 ayuntamientos de la provincia de Albacete, para comprobar si su Corporación Local poseía sede web oficial, es decir, para que confirmaran si su URL es oficial o si al menos se hallaba en proceso de construcción.

4. Se realizó una primera revisión de los 87 ayuntamientos y se encontró que todos disponía de una URL consistente en un subdominio dentro de un servidor web institucional, es decir, dentro de la URL de la Diputación de Albacete (http://www.dipualba.es/Main/) donde encontramos datos de contacto y dirección de cada Ayuntamiento. Además, algunos ayuntamientos también tenían URL oficial. 
La gran mayoría de las URL encontradas en un primer momento no eran oficiales, pues las había elaborado alguien del municipio.

5. AVANZA LOCAL. Se realizó una segunda revisión de los 87 ayuntamientos, observándose que hay corporaciones municipales que no tenían portal web oficial y ni siquiera estaba en construcción. Sin embargo, también se observó que la gran mayoría estaba construyendo su web oficial. Además, se comprobó que algunas de las sedes web oficiales aún no estaban operativas por encontrarse en un estado incipiente de construcción y desarrollo.

Así, se descartó tanto las que estaban en un estado inicial de construcción, como las que no eran oficiales o estaban en anteproyecto para ser construidas. A continuación se muestra un listado con las 64 webs evaluadas y la URL reservada para cada web:

1. Ayuntamiento de Albacete: http://www. albacete.es; http:/ / www.albacete.com

2. Ayuntamiento de Albatana: http://www. albatana.es

3. Ayuntamiento de Alborea: http://www. alborea.es

4. Ayuntamiento de Alcadozo: http://www. alcadozo.es

5. Ayuntamiento de Alcalá del Júcar: http:// www.alcaladeljucar.net

6. Ayuntamiento de Alcaraz: http://www. alcaraz.es

7. Ayuntamiento de Almansa: http://www. almansa.es

8. Ayuntamiento de Alpera: http://www. alpera.es

9. Ayuntamiento de Ayna: http://www. ayna.es

10. Ayuntamiento de Balazote: http://www. balazote.es

11. Ayuntamiento de Barrax: http://www. barrax.es

12. Ayuntamiento de Bienservida: http:// www.bienservida.es

13. Ayuntamiento de Bogarra: http://www. bogarra.es

14. Ayuntamiento de Bonete: http://www. bonete.es
15. Ayuntamiento de Carcelén: http://www. carcelen.es

16. Ayuntamiento de Casas de Juan Núñez: http://www.casasdejuannunez.es

17. Ayuntamiento de Casas de Lázaro: http:// www.casasdelazaro.es/

18. Ayuntamiento de Casas de Ves: http:// www.casasdeves.es

19. Ayuntamiento de Casas Ibáñez: http:// www.casasibañez.es;

http:/ / www.casasibanez.es/

20. Ayuntamiento de Caudete: http://www. caudete.es

21. Ayuntamiento de Cenizate: http://www. cenizate.es

22. Ayuntamiento de Chinchilla de Monte Aragón:

http:/ / www.chinchillademontearagon.com; http: / / chinchillademontearagon. sedelectronica.es/info.

23. Ayuntamiento de Corral Rubio: http:// www.corralrubio.es

24. Ayuntamiento de El Ballestero: http:// www.elballestero.es

25. Ayuntamiento de El Bonillo: http://www. elbonillo.es

26. Ayuntamiento de Elche de la Sierra: http:// www.elchedelasierra.es

27. Ayuntamiento de Férez: http://www. ferez.es

28. Ayuntamiento de Fuensanta: http://www. fuensanta.es

29. Ayuntamiento de Fuente Álamo: http:// www.aytofuentealamo.es/

30. Ayuntamiento de Fuentealbilla y Bormate: http://www.fuentealbilla.es/

31. Ayuntamiento de Golosalvo: http://www. golosalvo.es

32. Ayuntamiento de Hellín: http://www. hellin.org; http:/ / www.hellin.net

33. Ayuntamiento de Higueruela: http:// www.higueruela.es

34. Ayuntamiento de Hoya Gonzalo: http:// www.hoyagonzalo.es/

35. Ayuntamiento de La Gineta: http://www. lagineta.es

36. Ayuntamiento de La Roda: http://www. laroda.es

37. Ayuntamiento de Lezuza: http://www. lezuza.es

38. Ayuntamiento de Liétor: http://www. lietor.es 
39. Ayuntamiento de Madrigueras: http:// www.madrigueras.es

40. Ayuntamiento de Mahora: http://www. mahora.es

41. Ayuntamiento de Minaya: http://www. minaya.es

42. Ayuntamiento de Molinicos: http://www. molinicos.es/

43. Ayuntamiento de Montealegre del Castillo: http:/ / www.montealegredelcastillo.es

44. Ayuntamiento de Motilleja: http://www. motilleja.es

45. Ayuntamiento de Munera: http://www. munera.es

46. Ayuntamiento de Navas de Jorquera: http://www.navasdejorquera.es;

http:/ / www.dipualba.es/municipios/ navasjorquera

47. Ayuntamiento de Ontur: http://www.ontur.es

48. Ayuntamiento de Ossa de Montiel: http:// www.ossademontiel.es

49. Ayuntamiento de Peñas de San Pedro: http://www.peñasdesanpedro.es

50. Ayuntamiento de Pozo-Cañada: http:// www.pozocañada.es

51. Ayuntamiento de Pozohondo: http:// www.pozohondo.es/

52. Ayuntamiento de Pozo Lorente: http:// www.pozolorente.es

53. Ayuntamiento de Pozuelo: http://www. pozuelo.es/

54. Ayuntamiento de Riópar: http://www.riopar.es

55. Ayuntamiento de Robledo: http://www. robledo.es

56. Ayuntamiento de Salobre: http://www. salobre.es/s9d

57. Ayuntamiento de Socovos: http://www. socovos.es

58. Ayuntamiento de Tobarra: http://www.tobarra.es

59. Ayuntamiento de Valdeganga: http:// www.valdeganga.es

60. Ayuntamiento de Villa de Ves: http:// www.villadeves.es

61. Ayuntamiento de Villamalea: http://www. villamalea.es

62. Ayuntamiento de Villapalacios: http:// www.villapalacios.es

63. Ayuntamiento de Villarrobledo: http:// www.villarrobledo.es;

http:/ / www.villarrobledo.com;

64. AyuntamientodeVillaverdedeGuadalimar: http://www.villaverdedeguadalimar.es
Para la localización de las sedes web oficiales de los Ayuntamientos a evaluar fueron consultados:

- El directorio de ayuntamientos que ofrece el Ministerio de Hacienda y Administraciones Públicas:

- $\quad$ http://www.seap.minhap.es/index.html; http://www.seap.minhap.es/enlaces/ entidades_locales

- $\quad$ El directorio de ayuntamientos de la web 060.es:

- http://www.060.es/060/appmanager/ portal / desktop / page / portal_ page_251\&fia=portal_page_247

- $\quad$ El directorio de la Diputación de Albacete: http://www.dipualba.es

Tras eso se anotaron las URL de los ayuntamientos que ofrecían en dichos directorios y se realizaron búsquedas en el motor de búsqueda Google (http://www.google.com) para cada uno de los 87 municipios con dos objetivos: ver si existían otros ayuntamientos con presencia en la web que no estuvieran reflejados en los directorios anteriores y para los que sí estaban, comprobar si existían otros portales web municipales o relacionados con ellos.

\section{RESULTADOS Y DISCUSIÓN}

\section{I Resultados sobre el análisis de los dominios de primer nivel}

En la Tabla 10 se muestran los resultados sobre el análisis realizado con respecto a la distribución de dominios de primer nivel (".es", “.com", ".org", ".net", ".info", etc.) utilizados por los ayuntamientos de la provincia de Albacete en sus portales web. De la tabla se extrae que el 97,0\% del total de portales web analizados, disponen de un dominio ".es", es decir, 62 de las 64 web evaluadas. El 1,5\% utilizó el dominio ".net" y otro 1,5\% empleó el dominio ".org". El dominio ".com" y ".info" no fue utilizado por ningún ayuntamiento para denominar su web. Este porcentaje aumenta entre las webs de los ayuntamientos con menor número de habitantes, es decir, entre los municipios con menos de 5.001 habitantes. 
Tabla 10: Dominios de primer nivel distribuidos por número de población.

\begin{tabular}{|c|c|c|c|c|c|c|c|}
\hline \multicolumn{8}{|c|}{ DOMINIOS DE PRIMER NIVEL } \\
\hline \multirow{2}{*}{$\begin{array}{l}\text { TIPO DE MUNICIPIO } \\
\text { (Habitantes) }\end{array}$} & \multirow{2}{*}{$\begin{array}{c}\mathrm{N}^{\mathrm{o}} \text { de } \\
\text { Municipios }\end{array}$} & \multirow{2}{*}{$\begin{array}{c}\mathbf{N}^{o} \text { Total de } \\
\text { Municipios } \\
\text { Evaluados }\end{array}$} & \multicolumn{5}{|c|}{ DOMINIOS } \\
\hline & & & es & .com & .net & .org & info \\
\hline $\begin{array}{c}\text { Municipios con menos de } \\
5.001 \\
\end{array}$ & $\begin{array}{c}79 \\
(90,8 \%) \\
\end{array}$ & 57 & $\begin{array}{c}\mathbf{5 6} \\
(99,0 \%)\end{array}$ & $\begin{array}{c}\mathbf{0} \\
(0,0 \%)\end{array}$ & $\begin{array}{c}\mathbf{1} \\
(1,0 \%)\end{array}$ & $\begin{array}{c}\mathbf{0} \\
(0,0 \%)\end{array}$ & $\begin{array}{c}\mathbf{0} \\
(0,0 \%)\end{array}$ \\
\hline $\begin{array}{c}\text { Municipios entre } 5.001 \mathrm{y} \\
10.000 \\
\end{array}$ & $\begin{array}{c}2 \\
(2,3 \%) \\
\end{array}$ & 1 & $\begin{array}{c}1 \\
(100,0 \%) \\
\end{array}$ & $\begin{array}{c}\mathbf{0} \\
(0,0 \%) \\
\end{array}$ & $\begin{array}{c}\mathbf{0} \\
(0,0 \%) \\
\end{array}$ & $\begin{array}{c}\mathbf{0} \\
(0,0 \%) \\
\end{array}$ & $\begin{array}{c}\mathbf{0} \\
(0,0 \%) \\
\end{array}$ \\
\hline $\begin{array}{c}\text { Municipios entre } 10.001 \mathrm{y} \\
20.000\end{array}$ & $\begin{array}{c}2 \\
(2,3 \%)\end{array}$ & 2 & $\begin{array}{c}2 \\
(100,0 \%)\end{array}$ & $\begin{array}{c}\mathbf{0} \\
(0,0 \%)\end{array}$ & $\begin{array}{c}\mathbf{0} \\
(0,0 \%)\end{array}$ & $\begin{array}{c}\mathbf{0} \\
(0,0 \%)\end{array}$ & $\begin{array}{c}\mathbf{0} \\
(0,0 \%)\end{array}$ \\
\hline $\begin{array}{c}\text { Municipios entre } 20.001 \mathrm{y} \\
50.000 \\
\end{array}$ & $\begin{array}{c}3 \\
(3,5 \%) \\
\end{array}$ & 3 & $\begin{array}{c}2 \\
(99,0 \%) \\
\end{array}$ & $\begin{array}{c}\mathbf{0} \\
(0,0 \%) \\
\end{array}$ & $\begin{array}{c}\mathbf{0} \\
(0,0 \%)\end{array}$ & $\begin{array}{c}1 \\
(1,0 \%) \\
\end{array}$ & $\begin{array}{c}\mathbf{0} \\
(0,0 \%) \\
\end{array}$ \\
\hline Municipios con más de 50.000 & $\begin{array}{c}1 \\
(1,2 \%)\end{array}$ & 1 & $\begin{array}{c}\mathbf{1} \\
(100 \%) \\
\end{array}$ & $\begin{array}{c}\mathbf{0} \\
(0,0 \%) \\
\end{array}$ & $\begin{array}{c}\mathbf{0} \\
(0,0 \%) \\
\end{array}$ & $\begin{array}{c}\mathbf{0} \\
(0,0 \%)\end{array}$ & $\begin{array}{c}\mathbf{0} \\
(0,0 \%) \\
\end{array}$ \\
\hline TOTAL & $\begin{array}{c}87 \\
(100,0 \%)\end{array}$ & $\begin{array}{c}64 \\
(100,0 \%)\end{array}$ & $\begin{array}{c}62 \\
(97,0 \%)\end{array}$ & $\begin{array}{c}0 \\
(0,0 \%)\end{array}$ & $\begin{array}{c}1 \\
(1,5 \%)\end{array}$ & $\begin{array}{c}1 \\
(1,5 \%)\end{array}$ & $0(0,0 \%)$ \\
\hline
\end{tabular}

Fuente: Elaboración propia.

Asimismo, se comprobó cuáles son los ayuntamientos que habían reservado el dominio ".es" con su nombre. Para ello se realizó una consulta en la base de datos de ESNIC (https:// www.nic.es/sgnd/login.action), entidad responsable de la gestión del registro de nombres de dominio de Internet bajo el código de país ".es". Así pues, tras dicha consulta se pudo observar que casi todos los ayuntamientos evaluada ya habían reservado el dominio ".es", aunque todavía no se utilizara como URL oficial. Se comprobó que los 87 municipios tienen reservado el dominio “.es", es decir, el 100,0\%. Además, se advierte una tendencia a utilizar el topónimo del municipio, puesto que todas, excepto dos de las webs oficiales de los ayuntamientos evaluados, utilizan el dominio ".es", ".com" y ".org", y su URL es del siguiente tipo:
a. www.nombredelmunicipio.es;
b. www.nombredelmunicipio.com;
c. www.nombredelmunicipio.org.

Así, los resultados de la investigación revelan una tendencia a utilizar el dominio ".es" de primer nivel. Casi todos los ayuntamientos de la provincia de Albacete habían reservado el dominio "www.nombredelmunicipio.es", aunque todavía no se era utilizado en el momento de la evaluación (convendría que aquellos ayuntamientos que no utilicen el dominio, lo emplearan como una redirección de su URL)

\subsection{Resultados globales de la evaluación por grupos de indicadores}

A continuación se muestran los resultados obtenidos en la evaluación a través de cada uno de los grupos de indicadores seleccionados. Para facilitar la consulta de los datos obtenidos, dichos resultados se presentarán por grupo de servicios o indicadores. En los gráficos siguientes se muestra el número de cada indicador, y el porcentaje de sedes web oficiales analizadas que respondieron positivamente (SI) a cada indicador. Por tanto, se muestra el porcentaje web que respondieron "SI" al indicador sobre el total de webs evaluadas.

En el Gráfico 1 se indica el porcentaje total correspondiente al número de veces que cada indicador estaba presente en la evaluación, por ejemplo, 96,9\% respondió SI al indicador 1: ¿La autoría de la web está claramente definida en la página de Inicio o Home page?, es decir, dicho indicador fue evaluado positivamente ante la presencia de dicho 
indicador en 62 web analizadas, lo que supone el $96,9 \%$ (62 de 64 web evaluadas ofrecen el servicio al que hacer referencia el indicador 1$)$.

Lo más relevante de este grupo de indicadores es que el indicador número 5 (Indica cuándo ha sido la última actualización en la Home page) está presente en las páginas evaluadas con un $15,7 \%$, pues 10 de 64 webs evaluadas han respondido que SI ofrecen ese servicio. Otro aspecto a resaltar es que más de la mitad $(82,9 \%)$ de los ayuntamientos indica su dirección postal en la web (indicador 2). Asimismo, cabe destacar que el $92,2 \%$ de los ayuntamientos evaluados indican su email o webmaster en su portal web (indicador 4).

Gráfico 1: Aspectos Formales según cada indicador

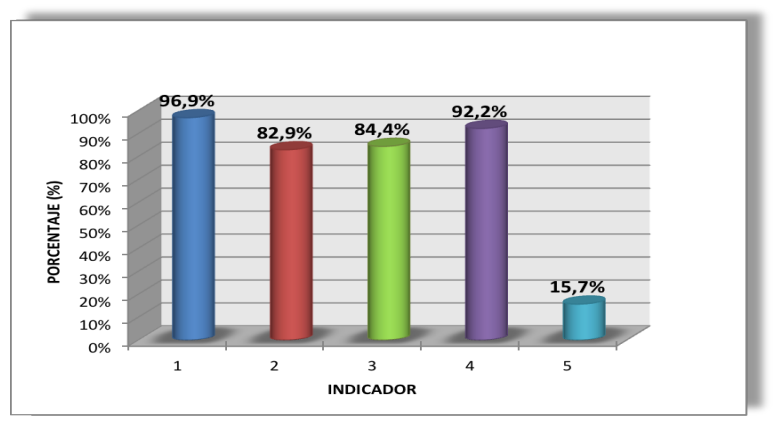

Fuente: Elaboración propia

En el Gráfico 2 se muestran los resultados obtenidos para el grupo de indicadores sobre Funcionalidad que permite evaluar la usabilidad, la facilidad de navegación, profundidad e información de ayuda a la navegación que ofrecen las diferentes webs municipales.

Gráfico 2: Funcionalidad según los indicadores

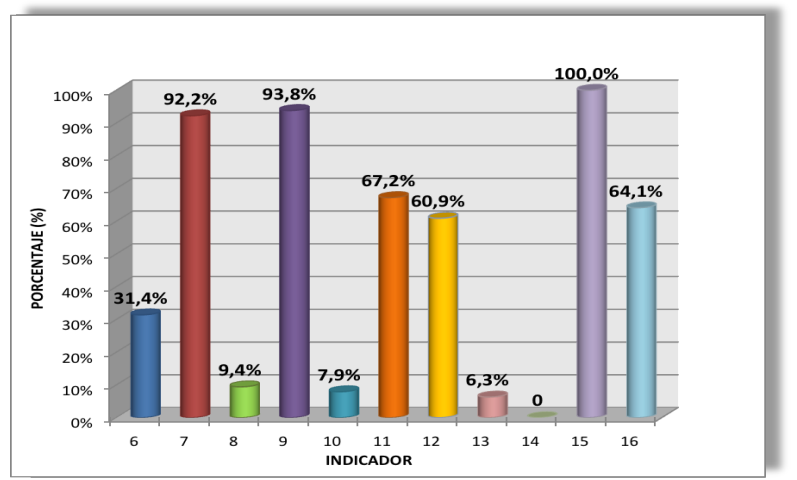

Fuente: Elaboración propia
Como se refleja en el Gráfico 2, el único indicador que alcanza el $100,0 \%$ de respuestas positivas es el indicador 15 (volver desde cualquier página a la página de inicio o home page), es decir, todos los ayuntamientos evaluados incluyen ese servicio. Los indicadores 7 (buscador interno) y 9 (enlaces a otras Administraciones) superan el 90,0\%. Los indicadores 11 (resolución de pantalla), 12 (navegadores para los que está optimizado) y 16 (menú "migas de pan") superan el 50,0\%. El resto se encuentra por debajo de este valor. Los indicadores 8 (versiones en otros idiomas), 10 (descarga de utilidades) y 13 (accesibilidad) están en niveles inferiores a lo deseable, pues no llegan el ni siquiera el $10,0 \%$ de webs que ofrecen esos servicios, a excepción del indicador 6 (mapa web) que supera 30,0\% de respuestas afirmativas. De alguna forma, los resultados obtenidos en estos indicadores revelan que algunas páginas web se hallan todavía en un estado incipiente de desarrollo y deben mejorar la usabilidad para facilitar la navegación por su portal web.

En cuanto al indicador número 14 (profundidad) se calculó el promedio y no la suma de respuestas positivas ante la presencia en la web de ese servicio. Por tanto, no procede hallar el porcentaje de la suma total de respuestas a este y aparece con el valor cero en el Gráfico 2. Así, se calculó el promedio del $\mathrm{n}^{\circ}$ de clics (profundidad de la web) y no la suma.

En la Tabla 11 se muestran tres columnas: el "Promedio del No de Clics" refleja el promedio de clics, el " $\mathrm{N}^{\circ}$ de Ayuntamientos" muestra el número de webs evaluadas que han respondido a ese promedio y la columna denominada "\% de Ayuntamientos" muestra el porcentaje total que representa la suma total de webs evaluadas. Como se puede observar, en la mayoría de las sedes web evaluadas $(86 \%)$ hay que pulsar como media unos 3 clics para acceder a la información ofrecida en el conjunto de las webs evaluadas, es decir, en 55 de 64 webs evaluadas hay que pulsar 3 clics como mínimo para acceder a la información deseada. 
Tabla 11: Profundidad en número de clics del indicador 14

\begin{tabular}{|c|c|c|}
\hline \multicolumn{3}{|c}{ PROFUNDIDAD (N DE CLICS) } \\
\hline $\begin{array}{c}\text { PROMEDIO DEL N } \\
\text { DE CLICS }\end{array}$ & N $^{\text {a }}$ DE AYUNTAMIENTOS & \% DE AYUNTAMIENTOS \\
\hline 2 & 2 & $3,0 \%$ \\
\hline 3 & 55 & $\mathbf{8 6 , 0} \%$ \\
\hline 4 & 7 & $\mathbf{1 1 , 0} \%$ \\
\hline TOTAL & 64 & $100 \%$ \\
\hline
\end{tabular}

Fuente: Elaboración propia

En el Gráfico 3 se muestran los resultados obtenidos en el grupo de indicadores que valora la información sobre el ayuntamiento ofrecida por los portales web analizados, es decir, muestran cómo se gestiona la información de carácter Institucional y los aspectos más representativos del Ayuntamiento.

En términos generales, los resultados obtenidos en este grupo son óptimos, aunque con algunas excepciones. El valor más elevado $(90,7 \%)$ pertenece al indicador 17 (Bienvenida del Alcalde/Alcaldesa). Por el contrario valor más bajo $(9,4 \%)$ corresponde a los indicadores 22 (Calendario fiscal municipal) y 23 (Presupuestos Municipales), unos servicios online importantes que los ayuntamientos deberían ofrecer a la ciudadanía, algo fundamental para favorecer la transparencia y el acceso a la información de la ciudadanía. Los ayuntamientos evaluados deberían ampliar dicha información, ya que por lo general es bastante reducida. Asimismo, cabe resaltar la puntuación obtenida en el indicador 20 (Actas de los Plenos Municipales), con un $40,7 \%$ de los ayuntamientos evaluados que ofrecen este servicio. Como vemos, es una cifra bastante baja, pues de 64 webs municipales evaluadas, solamente 26 ofrecían dicho servicio. Por último, destaca el resultado obtenido en el indicador 21 (Ordenanzas municipales), con un $42,2 \%$, una cifra bastante baja, ya que ni siquiera el $50,0 \%$ del total de webs evaluados incluyen esta información. No obstante, el número de ordenanzas que se puede consultar depende, en gran medida, del Ayuntamiento en cuestión.

Gráfico 3: Información sobre el Ayuntamiento según los indicadores

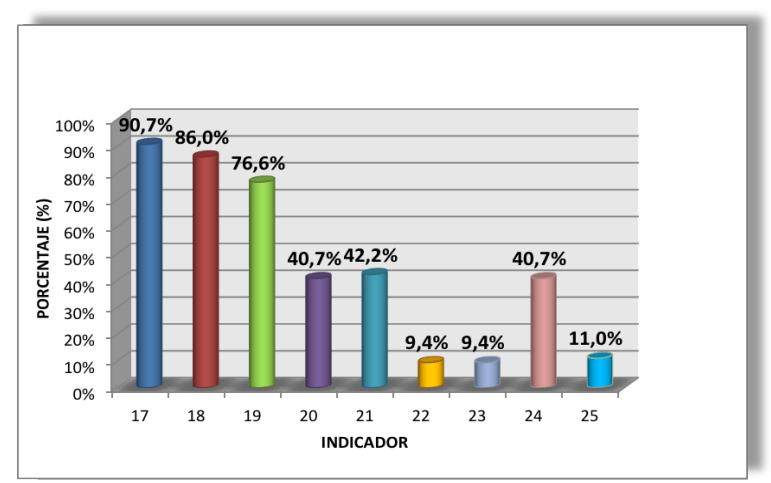

Fuente: Elaboración propia

En el Gráfico 4 se muestran los resultados obtenidos para los indicadores sobre la información del municipio disponible en la webs evaluadas. Los resultados obtenidos en este grupo son bastante positivos, ya que -a excepción del indicador 29 (incluye los principales datos estadísticos) que supone el 65,7\% -los demás están por encima del 90,0\%. Los indicadores que han logrado un mejor resultado son el 27 (historia del municipio) y el 28 (existencia de recursos multimedia), ambos con un $98,5 \%$. 
Gráfico 4: Información sobre la ciudad o municipio según los indicadores

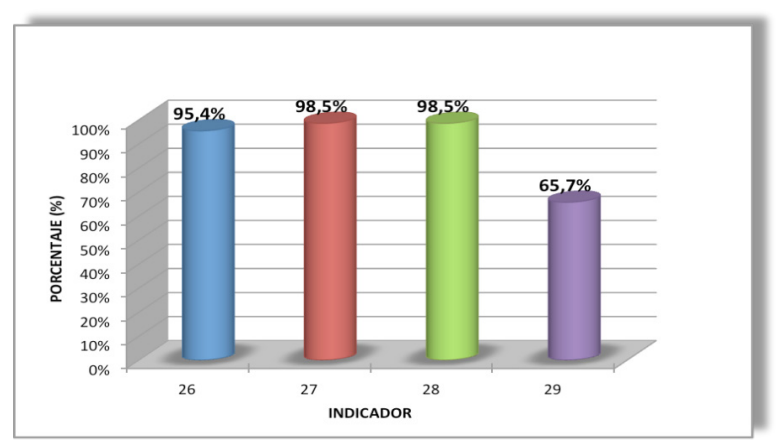

Fuente: Elaboración propia.

El Gráfico 5 refleja los resultados obtenidos para los indicadores que permiten medir el grado de información suministrado por los ayuntamientos a los ciudadanos para el desempeño habitual de sus labores diarias, de tal modo que la web municipal sea un lugar con información útil y actualizada para los vecinos del municipio.

Este grupo de indicadores no alcanza el 100,0\%, si bien los indicadores 30 (noticias de actualidad), 31 (agenda de actividades), 33 (directorio servicios de urgencia) y 38 (callejero de la localidad) superan el 90,0\%. De entre estos cuatro indicadores, cabe señalar que el 31 (agenda de actividades) representa el 90,7\%, lo que indica que la web se utiliza en algunos casos como un medio de transmisión de información desde los ayuntamientos hacia los ciudadanos.

Los indicadores 34 (directorio cultural), 36 (directorio Centros Sanitarios) y 39 (directorio de Asociaciones) superan el $30,0 \%$. El indicador 35 (directorio de Centros Educativos) y el 37 (información sobre el transporte público) supera el $20,0 \%$. El resto de indicadores, es decir, el indicador 32 (oficina de atención al ciudadano) y 40 (cuenta gratuita de correo electrónico) no alcanzan el $20,0 \%$ de presencia de estos servicios en las webs. Los indicadores (32 y 37) no tienen por qué existir en todos los municipios, por lo que es relativamente normal que sus cifras sean menores que las que alcanzan el resto de indicadores del grupo.
Gráfico 5: Información para los ciudadanos según los indicadores

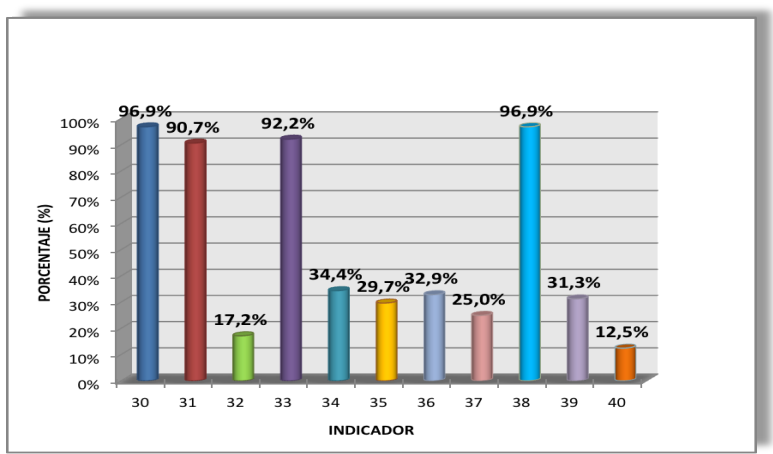

Fuente: Elaboración propia

En el Gráfico 6 se exponen los resultados obtenidos sobre los indicadores que permiten medir el grado de información turística y de ocio, tanto para los visitantes como para los propios ciudadanos del municipio, es decir, para residentes y no residentes. Se percibe que los indicadores 42 (directorio sobre bares, restaurantes), 43 (directorio de alojamientos de la localidad), 46 (guías turísticas, lugares de interés) y 47 (gastronomía y productos típicos) presentan valores que se encuentran por encima del $40,0 \%$ referente al porcentaje de web evaluadas que ofrecen dichos servicios, es decir, que han respondido Sí a esos indicadores. Únicamente el indicador 45 (fiestas y tradiciones locales) alcanza el 96,0\%. El indicadores 41 (cómo llegar) presenta un 39,1\% y el indicador 44 (oficina turismo), con un $21,9 \%$ de web con estos servicios.

Gráfico 6: Información Turística y de Ocio según los indicadores

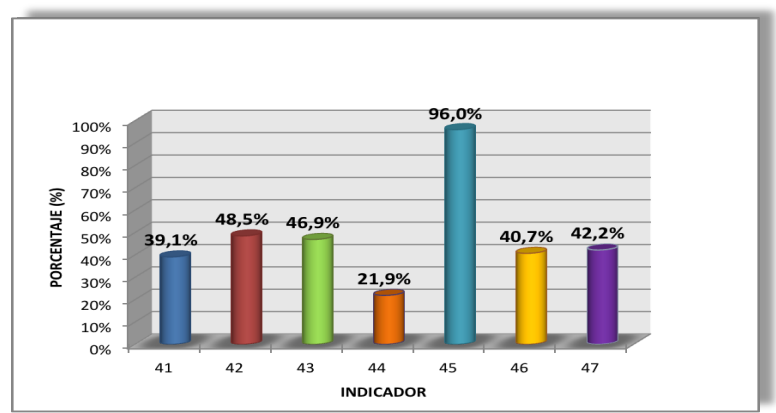

Fuente: Elaboración propia 
El Gráfico 7 muestra los resultados con respecto a los indicadores que permiten analizar la información sobre empresas y comercios de la localidad, la existencia de polígonos industriales y las ferias que se celebran en cada municipio. Cabe destacar el indicador 49 (información sobre el polígono industrial) con un resultado bastante óptimo $(62,5 \%)$ puesto que más del $50 \%$ de web evaluadas ofrecen ese servicio. El indicador 48 (directorio empresarial) representa el 31,3\% del total de sedes web evaluadas que responden Sí a ese indicador, pese a que es una información que tiende a cambiar constantemente y en algunos casos (en municipios de tamaño mediano-grande) resulta compleja su gestión, dado el volumen de información que genera. Además, existen otras herramientas y páginas web que proporcionan este tipo de información, tales como las guías telefónicas (páginas amarillas), guías de negocios, directorios sectoriales, etc.

Gráfico 7: Información sobre Empresas según los indicadores

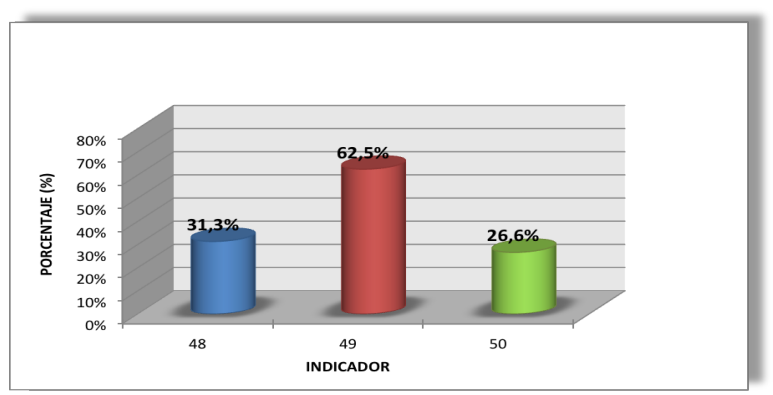

Fuente: Elaboración propia

Tal y como muestra el Gráfico 8, en el caso del indicador número 52 (respondió a la pregunta realizada: ¿Qué trámites debo realizar para darme de alta en el Padrón municipal?), el porcentaje de ayuntamientos que han respondido a la pregunta realizada, se realiza sobre el total de webs a las que se envió la pregunta (61 de 64 webs evaluadas). Así, se concluye que de los 64 ayuntamientos a los que se envió un correo electrónico preguntando por los trámites a realizar para poder darse de alta en el padrón municipal, respondieron 61, lo que supone el $95,4 \%$ del total de webs evaluadas a las que se envió dicha pregunta. $\mathrm{Al}$ respecto, cabe resaltar que los ayuntamientos que han respondido a la pregunta realizada, lo han hecho en un breve espacio de tiempo y todas las respuestas se pueden calificar de satisfactorias. Sin embargo, a 3 de las 64 webs evaluadas no se pudo enviar la pregunta por diversas causas: no tenía una dirección de correo, el formulario de contacto no funcionaba, no se obtuvo respuesta alguna ni siquiera automática, etc.

Gráfico 8: Servicios de Participación Ciudadana según los indicadores

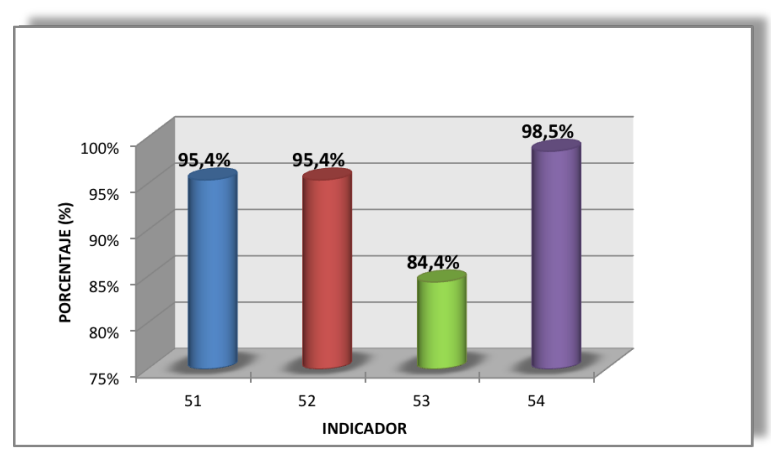

Fuente: Elaboración propia

De este gráfico también se desprende que el $84,4 \%$ de las webs evaluadas disponen de mecanismos de participación ciudadana, tales como encuestas, foros, etc., lo que supone un porcentaje relativamente alto. Otro dato significativo es que de los 64 ayuntamientos analizados, ofrecían el servicio de Buzón de quejas o sugerencia en sus sedes web 61, lo que supone el 95,4\%. Por último, el indicador 54 (solicitar cita previa) muestra el porcentaje más elevado y óptimo $(98,5 \%)$ de webs que ofrecen dicho servicio.

\section{CONSIDERACIONES FINALES}

A través de los resultados, ser percibe la gran importancia que tiene la visualización y el acceso a la información en la administración local, por ser la administración más cercana a los ciudadanos. Por otro lado, cabe señalar que se encontraron algunos enlaces dentro de las URL evaluadas que no funcionaban. Había páginas a las que no se podía acceder dentro de la misma web y que algunos portales web se cargaban 
mejor en Google Chrome y Mozilla Firefox, e Internet Explorer, por lo general, causaba más problemas en este aspecto.

No obstante, se observa que las Administraciones Públicas intentan incorporar las TIC, ya que lo ven como un medio para avanzar en el ámbito de la Administración electrónica. Asimismo, se percibe un esfuerzo por parte de los ayuntamientos por mejorar el diseño de sus webs y los servicios electrónicos ofrecidos a los ciudadanos. Sin embargo, estos esfuerzos aún no son suficientes, puesto que todavía queda un largo camino por recorrer en materia de $\mathrm{Ae}$ y transparencia pública de la información hasta alcanzar los parámetros marcados por la Unión Europea.

Por último, cabe señalar que se ha querido evitar cualquier tipo de matiz comparativo entre municipios, en cuanto a rangos o escalafones, ya que las condiciones particulares en lo referente al personal y equipamiento de cada Administración Local para ofrecer servicios TIC es difícilmente comparable con las demás. La presentación en tablas con puntos valorados binariamente (SI/ NO) facilitan la selección de los indicadores que deben o pueden ser susceptibles de mejora, haciendo comparaciones globales con el resto de municipios de la provincia albaceteña.

En definitiva, las Administraciones locales deben adoptar mecanismos eficientes que favorezcan la transparencia, la eficacia y una mayor cercanía entre la Administración y el ciudadano, es decir, una Ae orientada al ciudadano. Las AAPP han de permitir a la ciudadanía informarse e interactuar con sus ayuntamientos de forma electrónica (Internet) sin ninguna dificultad y evitando pasos innecesarios al usuario, facilitando el cumplimiento de las leyes de transparencia o derecho de acceso a la información del país. La instantaneidad cobra importancia en una sociedad marcada por un ritmo de vida acelerado y cambios vertiginosos, por lo que se hace imprescindible adaptarse a los nuevos cambios que van surgiendo y que implican el uso de las nuevas TIC en todos los ámbitos de nuestra realidad social. Así, las AAPP deben ofrecer servicios en línea e información de forma instantánea o inmediata durante las 24 horas del día, 365 días al año, permitiendo así, al ciudadano acceder a la información y servicios desde cualquier parte del mundo y en cualquier momento.

Artigo recebido em 2 I/ I0/2016 e aceito para publicação em 05/04/20I8

\section{TRANSPARÊNCIA AND ACESSO À INFORMAÇÃO SERVIÇO NOS SERVIÇOS ONLINE DOS SITES S DAS PREFEITURAS DE ALBACETE}

RESUMO

O objetivo desta pesquisa foi analisar o nível de consolidação e situação atual dos serviços eletrônicos oferecidos por 64 prefeituras da província de Albacete através de seus sites oficiais. Para este fim, foram definidas e selecionadas os serviços básicos online que as prefeituras devem oferecer aos cidadãos, seguindo as diretrizes estabelecidas no Plano de Ação da eEurope 2005 e do Plano de Ação i2010. Os dados foram coletados durante 12 meses, utilizando uma metodologia de avaliação dos sites e serviços online oferecidos pelas prefeituras avaliadas a partir de indicadores. Os instrumentos de coleta de dados foram a análise documental, questionários e observação experimental. Os resultados refletem os esforços das prefeituras para melhorar o design de seus sites e serviços electrónicos oferecidos aos cidadãos, embora ainda haja um longo caminho a percorrer para atingir os parâmetros fixados pela União Europeia.

Palavras-Chave: Administração eletrônica. Governo eletrônico. Administração local. Avaliação web. Sociedade da informação.

\section{REFERENCIAS}

COMISIÓN EUROPEA. La información del sector público: un recurso clave para Europa. Libro verde sobre la información del sector público en la sociedad de la información. Bruselas, 1998. Disponible en: $\quad<\mathrm{ftp}: / / \mathrm{ftp}$.cordis.europa.eu/pub/ econtent/docs/gp_es.pdf.> Consultado en: Enero 2011 
COMISIÓN EUROPEA. Plan de acción eEurope 2002. EUR-Lex, 2001. Disponible en: <http:// europa.eu/legislation_summaries/information_ society/strategies/124226a_es.htm> . Consultado en: noviembre 2011

COMISIÓN EUROPEA. Plan de acción eEurope 2005: una sociedad de la información para todos. Bruselas: EUR-Lex, 2002. Disponible en: <http:// europa.eu/legislation_summaries/information_ society/strategies/124226_es.htm> Consultado en: noviembre 2011

COMISIÓN EUROPEA. Plan de acción sobre administración electrónica i2010: acelerar la administración electrónica en Europa en beneficio de todos. Bruselas: EUR-Lex, 2006. Disponible en: <http://europa.eu/legislation_summaries/ information_society/strategies/124226j_es.htm> Consultado en: noviembre 2011

EUROPEAN COMMISSION (EC) i2010 - A European Information Society for growth and employment. Communication from the Commission to the Council, the European Parliament, the European Economic and Social Committee and the Committee of the Regions. Brussels, 2005.

FUNDACIÓN AUNA: e-España 2005. Fundación AUNA. Madrid. 2005.

MINISTERIO DE ADMINISTRACIONES PÚBLICAS (MAP) Ley 11/2007, de 22 de junio, de Acceso Electrónico de los Ciudadanos a los Servicios Públicos (LAECSP). 2007. Disponible en: $\quad$ http: <//www.boe.es/diario_boe/txt. php?id=BOE-A-2007-12352>. Consultado en: diciembre 2011

MOYA MARTÍNEZ, G. La Administración Pública en línea: estudio sobre los servicios públicos de los ayuntamientos de la región de Murcia basados en Web. Anales de Documentación, n.6 pp.187-202, 2003

MOYA MARTÍNEZ, G., RODRÍGUEZ MUÑOZ, J. V., MARTÍNEZ MÉNDEZ, F. J.; DÍAZ ORTUÑO, P.M. Análisis de las webs municipales de la Región de Murcia. Diciembre 2005. Fundación Integra, 2005.
Disponible en: <http://www.regmurcia.com/ servlet/s.Sl? sit=c,364\&r=ReP-10768-DETALLE_ REPORTAJES>. Consultado en: Mayo 2011

MOYA MARTÍNEZ, G.; RODRÍGUEZ MUÑOZ, J. V.; MARTÍNEZ MÉNDEZ, F. J.; DÍAZ ORTUÑO, P.M. Análisis de las webs municipales de la Región de Murcia. Diciembre 2006. Fundación Integra, 2006. Disponible en: <http://www.regmurcia.com/ servlet/s.Sl? sit=c,364\&r=ReP-16234-DETALLE_ REPORTAJES.> Consultado en Mayo 2011

Análisis de las webs municipales de la Región de Murcia 2008. Mayo 2009. Fundación Integra, 2009. Disponible en: <http://www. regmurcia.com/servlet/s.Sl sit $=c, 364 \& r=R e P-$ 25162-DETALLE_REPORTAJES>. Consultado en: Mayo 2011

Análisis de las webs municipales de la Región de Murcia 2010. Febrero 2011. Fundación Integra, 2010. Disponible en: <http:/ / www.regmurcia. com/servlet/s.Sl?sit=c,364\&r=ReP-27831-DETALLE_ REPORTAJES>. Consultado en: Mayo 2011

ORGANIZATION FOR ECONOMIC COOPERATION AND DEVELOPMENT (OECD) Policy Brief "The e-Government Imperative: Main Findings". OCDEobserver. París, 2003. Disponible en: <http://unpan1.un.org/ intradoc/groups/public/documents/APCITY/ UNPAN015120.pdf. > Consultado en: Mayo 2011

ORGANIZATION FOR ECONOMIC COOPERATION AND DEVELOPMENT (OECD/ OCDE) e-Government for better government. OECDpublishing, 2005. Traducción: INAP (Teodoro Sacristán), e-Gobierno para un mejor gobierno. INAP, 2009.

SI-LOC@L.1er Análisis de las páginas web de los ayuntamientos asturianos. 2002. Disponible en: <http://www.silocal.org/aytos/www_01. htm>. Consultado en: septiembre 2011

SUREDA NEGRE, J.; COMAS FORGAS, R. Els ajuntaments de les Illes Balears a Internet. Primera anàlisi de les webs municipals de les Illes Balears i descripció d'una sèrie de bones practiques. 2004. 
WEST, D. M. E-Government and the Transformation of Service Delivery and Citizen Attitudes. Public Administration Review. 2004.

WEST, D. Urban e-government 2003. Septiembre 2003. Disponible en:< http:/ / www.insidepolitics. org/egovt03city.html >. Consultado en: diciembre 2011 